\title{
Analysis of Chaotic Ferroresonance Phenomena in Unloaded Transformers Including MOV
}

\author{
Ataollah Abbasi ${ }^{1}$, Mehrdad Rostami ${ }^{1}$, Ahmad Gholami ${ }^{2}$, Hamid R. Abbasi ${ }^{2}$ \\ ${ }^{1}$ Shahed University, Tehran, Iran \\ ${ }^{2}$ Iran University of Science and Technology, Tehran, Iran \\ E-mail: \{abbasi, Rostami\}@shahed.ac.ir,gholami@iust.ac.ir, hamid2005444@yahoo.com \\ Received June 5, 2011; revised July 15, 2011; accepted July 23, 2011
}

\begin{abstract}
We study the effect of a parallel metal oxide surge arrester on the ferroresonance oscillations of transformers. It is expected that the arresters generally cause ferroresonance drop out. Simulation has been done on a three phase power transformer with one open phase. Effect of varying input voltage is studied. The simulation results reveal that connecting the arrester to transformers poles, exhibits a great mitigating effect on ferroresonant over voltages. Phase plane along with bifurcation diagrams are also presented. Significant effect on the onset of chaos, the range of parameter values that may lead to chaos and magnitude of ferroresonant voltages is obtained, shown and tabulated.
\end{abstract}

Keywords: Power Transformer, Phase Plane Diagram, Bifurcation Diagram, Chaotic Ferroresonance

\section{Introduction}

Ferroresonance is a complex nonlinear electrical phenomenon that can cause dielectric \& thermal problems to components power system. Electrical systems exhibiting ferroresonant behaviour are categorized as nonlinear dynamical systems. Therefore conventional linear solutions cannot be applied to study ferroresonance. The prediction of ferroresonance is achieved by detailed modeling using a digital computer transient analysis program [1]. Ferroresonance should not be confused with linear resonance that occurs when inductive and capacitive reactance of circuit is equal. In linear resonance the current and voltage are linearly related and are frequency dependent. In the case of ferroresonance it is characterized by a sudden jump of voltage or current from one stable operating state to another one. The relationship between voltage and current is depends not only on frequency but also on other factors such as system voltage magnitude, initial magnetic flux condition of transformer iron core, total loss in the ferroresonant circuit and moment of switching [2].

Ferroresonance may be initiated by contingency switching operation, routine switching, or load shedding involving a high voltage transmission line. It can result in Unpredictable over voltages and high currents. The prerequisite for ferroresonance is a circuit containing iron core inductance and a capacitance. Such a circuit is characterized by simultaneous existence of several steadystate solutions for a given set of circuit parameters. The abrupt transition or jump from one steady state to another is triggered by a disturbance, switching action or a gradual change in values of a parameter. Typical cases of ferroresonance are reported in [1-4]. Theory of nonlinear dynamics has been found to provide deeper insight into the phenomenon. [5-8] are among the early investigations in applying theory of bifurcation and chaos to ferroresonance. The susceptibility of a ferroresonant circuit to a quasi-periodic and frequency locked oscillations are presented in $[9,10]$. The effect of initial conditions is also investigated. The effect of transformer modeling on the predicted ferroresonance oscillations is studied in [11]. Using a linear model, authors of [12] have indicated the effect of core loss in damping ferroresonance oscillations. The importance of treating core loss as a nonlinear function of voltage is highlighted in [7]. An algorithm for calculating core loss from no-load characteristics is given in [13]. Evaluation of chaos in voltage transformer, effect of resistance of key on the chaotic behavior voltage transformer and subharmonics that produced with ferroresonance in this type transformer and quantification of the chaotic behavior of ferroresonant voltage transformer circuits are studied in $[9,14,15]$. 


\section{System Modeling}

Transformer is assumed to be connected to the Power System while one of the three switches are open and only two phases of it are energized, which produces induced voltage in the open phase. This voltage, back feeds the distribution line. Ferroresonance will occur if the distribution line is highly capacitive. System involves the nonlinear magnetizing reactance of the transformer's open phase and resulted shunt and series capacitance of the distribution line.

Base system model is adopted from [3] with the MOV arrester connected across the transformer winding which is showed in Figure 1 Linear approximation of the peak current of the magnetization reactance can be presented by Equation (1):

$$
i_{l}=a \lambda
$$

However, for very high currents, the iron core might be saturated where the flux-current characteristic becomes highly nonlinear. The $\lambda-i_{l}$ characteristic of the transformer can be demonstrated by the polynomial in Equation (2):

$$
i_{l}=a \lambda+b \lambda^{q}
$$

Arrester can be expressed by the Equation (3):

$$
V=K I^{\alpha}
$$

$V$ represents resistive voltage drop, $I$ represents arrester current and $K$ is constant and $\alpha$ is nonlinearity constant. The differential Equation for the circuit in Figure 1 can be derived as follows:

$$
\begin{aligned}
\omega E \cos \omega t & =p^{2} \lambda+\frac{p \lambda}{R C}+\left(\frac{1}{C}\right)\left(a \lambda+b \lambda^{q}\right) \\
& +\left(\frac{1}{C}\right)\left(\frac{|p \lambda|}{K}\right)^{\alpha} \operatorname{sign}(p \lambda)
\end{aligned}
$$

where $p=\frac{d}{d t}$ and $\omega$ represents the power frequency and $E$ is the peak value of the voltage source, shown in Figure 1.

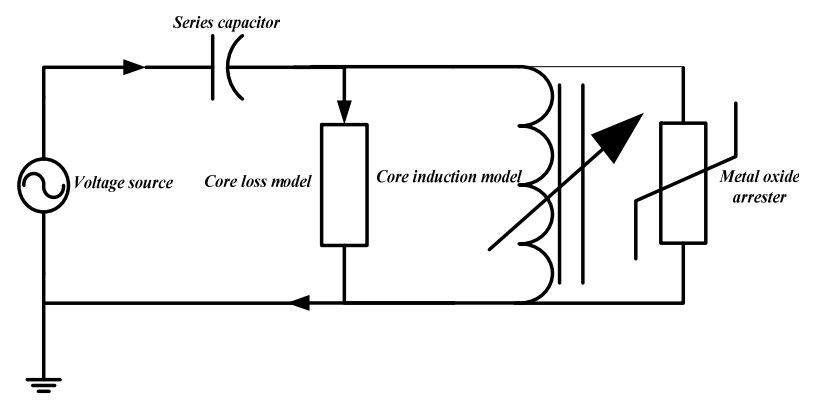

Figure 1. Circuit of system.
Presenting in the form of state space Equations, $\lambda$ and $p \lambda$ will be state variables as follows:

$$
\begin{gathered}
\lambda=x_{1}, p \lambda=x_{2} \\
\dot{x}_{1}=x_{2} \\
\dot{x}_{2}=\omega E \cos \omega t-\frac{x_{2}}{R C}-\left(\frac{1}{C}\right)\left(a x_{1}+b x_{1}^{q}\right) \\
-\left(\frac{1}{C}\right)\left(\frac{\left|x_{2}\right|}{K}\right)^{\alpha} \operatorname{sign}\left(x_{2}\right)
\end{gathered}
$$

\section{Simulation Results}

Typical values for various system parameters considered for simulation are as given below [5]:

$$
\begin{gathered}
q=5 \rightarrow\left\{\begin{array}{l}
b=0.0005 \\
a=0
\end{array}\right. \\
q=7 \rightarrow\left\{\begin{array}{l}
b=0.001 \\
a=0
\end{array}\right. \\
q=11 \rightarrow\left\{\begin{array}{l}
b=0.0072 \\
a=0.0028 ;
\end{array}\right. \\
\omega=1 \text { p.u., } R=100 \text { p.u., } C=0.047 \text { p.u. } \\
E=0 \text { - } 6 \text { p.u., } K=2.501, \alpha=25 .
\end{gathered}
$$

\begin{tabular}{|c|c|c|c|}
\hline $\mathrm{q}$ & 1 & 2 & 3 \\
\hline 5 & Priodic & Priodic & Priodic \\
\hline 7 & Priodic & Priodic & Chaotic \\
\hline 11 & Priodic & Priodic & Chaotic \\
\hline \multicolumn{4}{|c|}{ (b) } \\
\hline$q$ & 4 & 5 & 6 \\
\hline 5 & Chaotic & Chaotic & Chaotic \\
\hline 7 & Chaotic & Chaotic & Chaotic \\
\hline 11 & Chaotic & Chaotic & Chaotic \\
\hline
\end{tabular}

Initial conditions:

$$
\lambda(0)=0, p \lambda(0)=1.44 \text { p.u. }
$$

Table 1 shows different values of $E$, considered for analyzing the circuit in absence of surge arrester.

Table 2 includes the set of cases which are considered for analyzing the circuit including arrester:

Time domain simulations were performed using the MATLAB programs which are similar to EMTP simulation [3]. For cases including arrester, it can be seen that

Table 1. (a) Behaviour of system without MOV for $E=1,2$, 3. (b) Behaviour of system without MOV for $E=4,5,6$.

(a) 
Table 2. (a) Behaviour of system with MOV for $E=1,2,3$. (b) Behaviour of system with MOV for $E=4,5,6$.

(a)

\begin{tabular}{|c|c|c|c|}
\hline $\mathrm{q}$ & 1 & 2 & 3 \\
\hline 5 & Priodic & Priodic & Priodic \\
\hline 7 & Priodic & Priodic & Priodic \\
\hline 11 & Priodic & Chaotic & Priodic \\
\hline
\end{tabular}

(b)

\begin{tabular}{|c|c|c|c|}
\hline $\mathrm{q}$ & 4 & 5 & 6 \\
\hline 5 & Priodic & Priodic & Priodic \\
\hline 7 & Priodic & Priodic & Chaotic \\
\hline 11 & Chaotic & Chaotic & Chaotic \\
\hline
\end{tabular}

ferroresonant drop out will be occurred.

Figure 2 show the phase plane plot of system states without arrester for $E=1$ p.u.

Figure 3 shows the phase plane plot and time domain simulation of system states without arrester for $E=4$ p.u. which depicts chaotic behavior and Figure 4 shows the corresponding time domain wave form.

Also Figures 5-7 show the bifurcation diagram of chaotic behaviours for three of values of $q$. The system shows a greater tendency for chaos for saturation characteristics with lower knee points, which corresponds to higher values of exponent $q$.

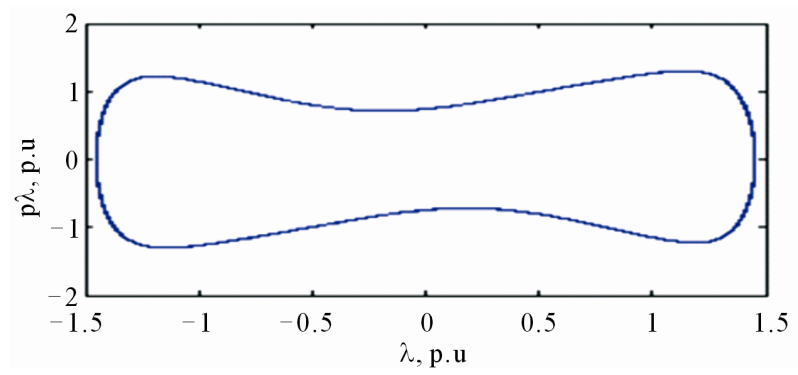

Figure 2. Phase plane diagram for $E=1, q=11$ without MOV.

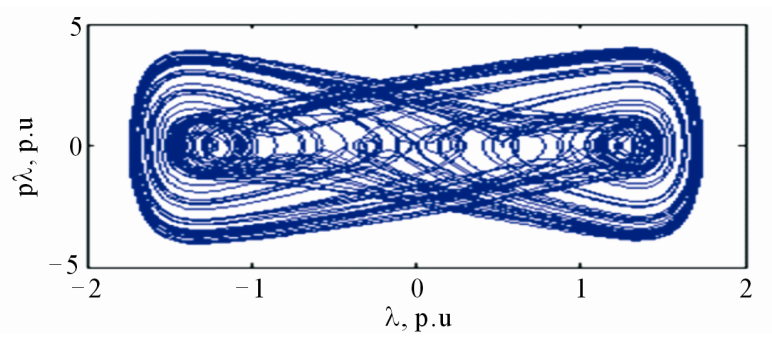

Figure 3. Phase plane diagram for $E=4, q=11$ without MOV.

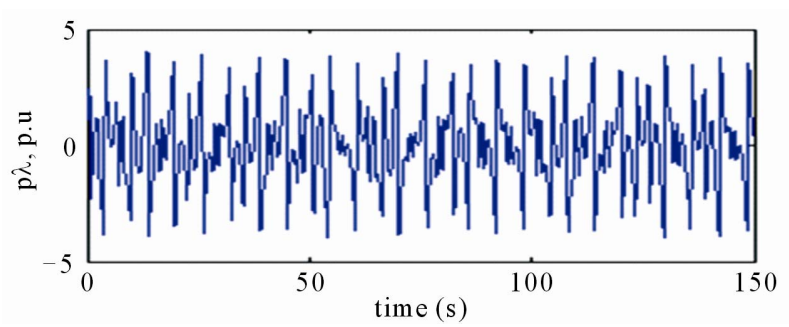

Figure 4. Time domain chaotic wave form for $E=4, q=11$ without MOV.

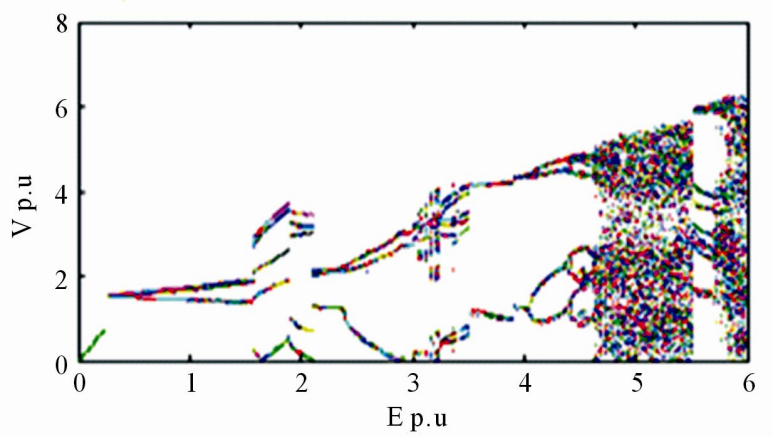

Figure 5. Bifurcation diagram for $q$ = without MOV.

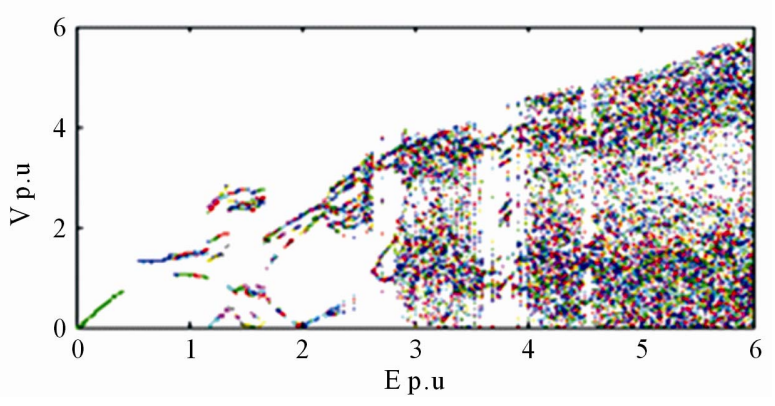

Figure 6. Bifurcation diagram for $q=7$ without MOV.

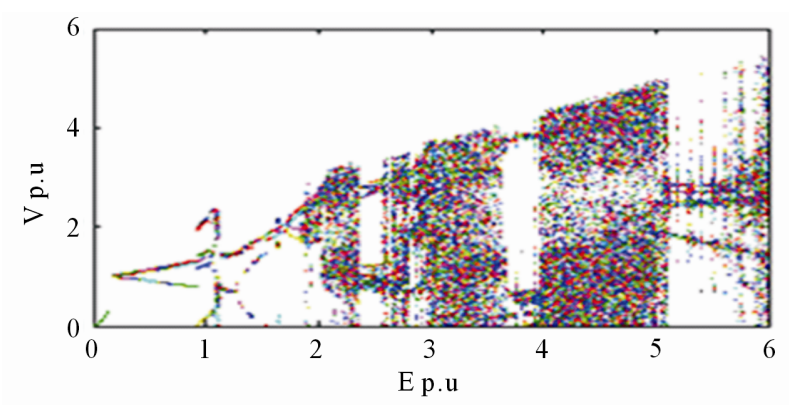

Figure 7. Bifurcation diagram for $q=11$ without MOV.

Figures 8-10 show that chaotic region mitigates by applying MOV surge arrester. Tendency to chaos exhibited by the system increases while $q$ increases too.

With consideration to Figures 8-10 MOV makes a mitigation in ferroresonance chaotic behavior in transformer that in down value of $q$ the chaotic region are 


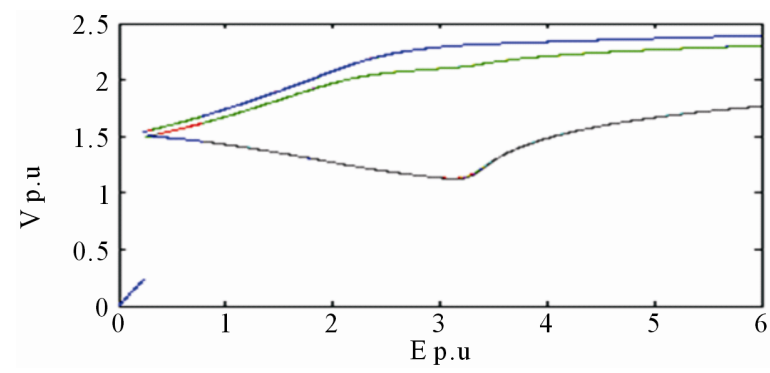

Figure 8. Bifurcation diagram for $q=5$ with $\mathrm{MOV}$.

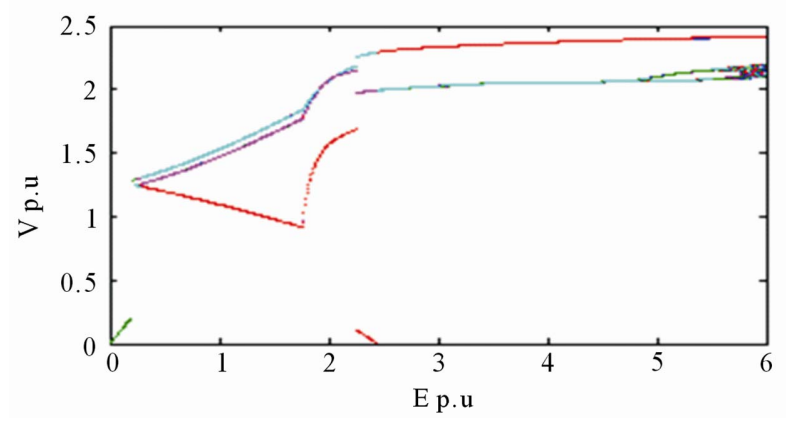

Figure 9. Bifurcation diagram for $q=7$ with MOV.

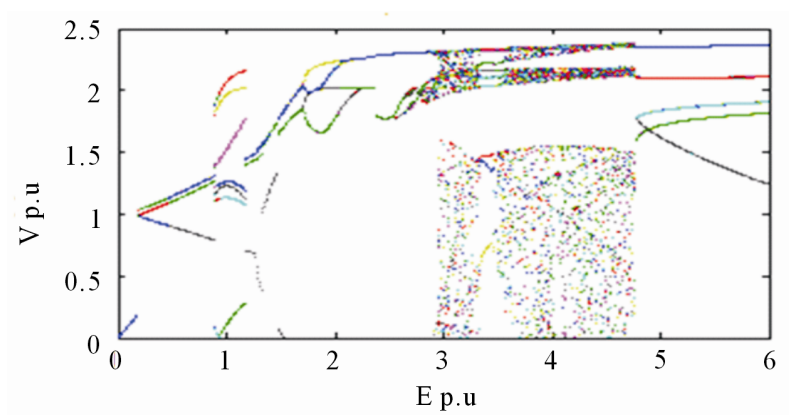

Figure 10. Bifurcation diagram for $q=11$ with MOV.

removed and the behavior will be periodic, for greater value of $q$ for example for $q=11$ independent chaotic regions which can be created under MOV nominal voltage have survived so chaotic behavior has been eliminated.

\section{Conclusions}

The presence of the arrester results in clamping the Ferroresonant over voltages in the studied system. The arrester successfully suppresses or eliminates the chaotic behaviour of proposed model. Consequently, the system shows less sensitivity to initial conditions in the presence of the arrester. It is seen from the bifurcation diagram that chaotic ferroresonant behavior depends on parameter q. MOV makes a mitigation in ferroresonance chaotic behavior in transformer that in down value of $q$ the chaotic region are removed and the behavior will be periodic.
System stability increased with decreasing $q$ and chaotic regions are eliminiated. It is found when $q=11$ at $v_{\text {in }}=4$ p.u. beahavior of system is chaotic while for $q=7$ in the same value of $v_{i n}$ system is in subharmonic mode and its stability is more than case that $q=11$.

\section{References}

[1] Z. Emin and K. Y. Tong, "Ferroresonance Experience in UK: Simulations and Measurements," International Conference on Power Systems Transients (IPST), Rio de Janeiro, 24-28 June 2001.

[2] R. N. Mukerjee, B. Tanggawelu, E. A. Ariffin and M. Balakrishnan, "Indices for Ferroresonance Performance Assessment in Power Distribution Network," International Conference on Power Systems Transients (IPST), New Orleans, 28 September-2 October, 2003.

[3] S. Mozaffari, M. Sameti and A. C. Soudack, "Effect of Initial Conditions on Chaotic Ferroresonance in Power Transformers," IEE Proceedings/Generation, Transmission and Distribution, Vol. 144, No. 5, 1997, pp. 456460. doi:10.1049/ip-gtd:19971459

[4] K. Al-Anbarri, R. Ramanujam, R. Saravanaselvan and K. Kuppusamy, "Effect of Iron Core Loss Nonlinearity on Chaotic Ferroresonance in Power Transformers,” Electric Power Systems Research Elsevier Journal, Vol. 65, No. 1, 2003, pp. 1-12.

[5] K. Pattanapakdee and C. Banmongkol, "Failure of Riser Pole Arrester Due to Station Service Transformer Ferroresonance," International Conference on Power Systems Transients (IPST), Lyon, 4-7 June 2007.

[6] A. Abbasi, M. Rostami, H. Radmanesh and H. Abbasi, "Elimination of Chaotic Ferroresonance in Power Transformers Including Nonlinear Core Losses Applying of Neutral Resistance," Environment and Electrical Engineering 2009, IEEE Conference, Wroclaw, 10-13 May 2009.

[7] Z. A. Rezaei, P. M. Sanaye, H. Mohseni, S. Farhangi and R. Iravani, "Analysis of Ferroresonance Modes in Power Transformers Using Preisach-Type Hysteretic Magnetizing Inductance,” IEEE Transactions on Power Delivery, Vol. 22, No. 2, April 2007, pp. 919-929. doi:10.1109/TPWRD.2006.877078

[8] A. Abbasi, M. Rostami, H. Radmanesh and H. R. Abbasi, "Evaluation of Chaotic Ferroresonance in Power Transformers including Nonlinear Core Losses,” Environment and Electrical Engineering 2009, IEEE Conference, Wroclaw, 10-13 May 2009.

[9] A. E. A. Araujo, A. C. Soudack and J. R. Marti, "Ferroresonance in Power Systems: Chaotic Behaviour," IEE Proceedings Part C, Vol. 140, No. 3, May 1993, pp. 237-240.

[10] S. K. Chkravarthy and C. V. Nayar, "Frequency-Locked and Quasi Periodic (QP) Oscillations in Power Systems," IEEE Transactions on Power Delivery, Vol. 13, No. 2, 1997, pp. 560-569. doi:10.1109/61.660929

[11] B. A. Mork, "Five-Legged Wound/Core Transformer Model: 
Derivation, Parameters, Implementation, and Evaluation,” IEEE Transactions on Power Delivery, Vol. 14, 1999, pp. 1519-1526. doi:10.1109/61.796249

[12] Z. A. Rezaei, H. Mohseni, P. M. Sanaye, S. Farhangi and R. Iravani, "Performance of Various Magnetic Core Models in Comparison with the Laboratory Test Results of a Ferroresonance Test on a 33 KV Voltage Transformer”, IEEE Power Engineering Society General Meeting, Montreal, 16 October 2006.

[13] W. L. A. Neves and H. Dommel, "On Modeling Iron Core Nonlinearities,” IEEE Transactions on Power Sys- tems, Vol. 8, No. 2, 1993, pp. 417-425. doi:10.1109/59.260845

[14] G. Kavasseri, "Analysis of Subharmonic Oscillations in a Ferroresonant Circuit,” Electrical Power and Energy Systems, Elsevier Journal, Vol. 28, No. 3, 2005, pp. 207214.

[15] Z. Emin, B. A. T. Zahawi, Y. K. Tong and M. Ugur, "Quantification of the Chaotic Behavior of Ferroresonant Voltage Transformer Circuits," IEEE Transactions on Circuits and Systems, Fundamental Theory and Applications, Vol. 48, No. 6, June 2001, pp. 757-760. 